\title{
Cemented versus Uncemented Partial Knee Arthroplasty Trial - The CUPKAT Trial - A prospective, randomized controlled trial
}

\section{Benjamin Panzram}

Center for Orthopedics, Trauma Surgery and Spinal Cord Injury, Ruprecht-Karls Universität Heidelberg https://orcid.org/0000-0001-9113-2882

\section{Christian Merle}

Center for Orthopedics, Trauma Surgery and Spinal Cord Injury, Ruprecht-Karls Universität Heidelberg

Christoph Rehnitz

Clinic of Diagnostic and Interventional Radiologie, Ruprecht-Karls Universität Heidelberg

\section{Andrea Seidel-Glätzer}

Coordination Center for Clinical Studies, Ruprecht-Karls Universität Heidelberg

\section{Thomas Bruckner}

Institut of Medical Biometry and Informatics, Ruprecht-Karls Universität, Heidelberg

\section{Tobias Gotterbarm}

Department for Orthopedics and Traumtology, Kepler University, Linz

\section{Tobias Reiner ( $\boldsymbol{\sim}$ Tobias.Reiner@med.uni-heidelberg.de )}

Center for Orthopedics, Trauma Surgery and Spinal Cord Injury, Ruprecht-Karls Universität Heidelberg

\section{Study protocol}

Keywords: Cementless UKA, OUKRA, radiolucent lines, radiolucencies, cementless fixation, Oxford medial

Posted Date: July 28th, 2020

DOI: https://doi.org/10.21203/rs.3.rs-28953/v1

License: (c) (1) This work is licensed under a Creative Commons Attribution 4.0 International License. Read Full License 


\section{Abstract}

\section{Background}

Cemented Oxford unicompartmental knee arthroplasty (OUKA) has shown excellent clinical and functional long-term results as a treatment option for patients with end-stage anteromedial osteoarthritis of the knee. Despite the good long-term results that have been reported in the literature, registry data show that aseptic loosening and pain associated with the appearance of commonly seen radiolucencies around the tibial component are the most common causes for revision surgery in cemented OUKA. In order to address these problems, the cementless OUKA has been developed. Initial studies indicate excellent clinical short-term results for cementless OUKA although the risk of tibial head fracture seems to be elevated and long-term results are not yet available. Given the ongoing debate about the benefits or disadvantages of cementless fixation in knee arthroplasty, there is a need for independent level I and level II studies in order to assess the safety and equivalence of cementless OUKA in comparison to the welldocumented clinical results of the cemented OUKA.

\section{Methods/Design}

This study has a prospective, randomized, controlled, double-centre, non-inferiority design. A total of 306 Patients with anteromedial OA will be randomized to cementless or cemented OUKA either at the Heidelberg University Hospital or at the Kepler University Hospital Linz. As primary outcome measure we evaluate the Oxford Knee Score (OKS) to compare clinical and functional results 2 years after cemented versus uncemented OUKA.

Secondary outcome measures include clinical and functional scores as measured with the American Knee Society Score (AKSS), UCLA Activity Score, Knee Injury and Osteoarthritis Outcome Score (KOOS), Forgotten Knee Score (FJS-12), Visual Analog Pain Score (VAS), and health related quality of life questionaire (EQ-5D). In addition, complications such as the occurrence of periprosthetic fractures or implant loosening, incidence of radiolucencies and reoperation rates among others will be evaluated as secondary outcome measures and compared between the two groups. A subgroup of patients $(n=150)$ will be asked to undergo additional radiographic DXA measurement to assess the changes in bone mineral density around the femoral and tibial components preoperatively and two years after surgery.

\section{Trials Registration}

Prior to the beginning of the clinical phase (FPI) we registered the trial at Deutsches Register Klinischer Studien (German Clinical Trials Register) with the registration number: DRKS00021303. Registered 30 April 2020. The Registration will be forwarded to the to the WHO within the next update (within the next 4 weeks) and will be searchable via its Meta-registry ( http://apps.who.int/trialsearch/ ).

\section{Background}


Oxford unicompartmental knee arthroplasty (OUKA) has shown excellent clinical and functional longterm results as a treatment option for patients with end-stage anteromedial osteoarthritis of the knee [14]. Though good long-term results have been reported in the literature, registry data demonstrate that the revision rate after unicompartmental knee arthroplasty (UKA) is higher compared to total knee arthroplasty [5]. Among others, progression of osteoarthritis, deep wound infection, inlay dislocation or persisting pain are possible reasons for revision surgery [6-8]. However, the most frequent reason for revision surgery in UKA is aseptic loosening which accounts for up to $46 \%$ of all revisions $[9,10]$.

A well-known phenomenon, especially in cemented UKA is the occurrence of radiolucencies at the cement-bone interface of the tibial component [11]. The most common radiolucent line is usually $2 \mathrm{~mm}$ or less thick and has a sclerotic margin. In contrast to pathological radiolucencies, associated with implant loosening, it tends to consolidate during the first postoperative year and is not associated with significant problems or inferior clinical outcome [11]. These "physiological" radiolucencies have been reported to be observed in $60 \%$ to $97 \%$ of knees with a cemented Oxford unicompartmental knee arthroplasty [12]. However, if they occur in the context of unexplained pain, they might raise concerns and be the cause of revision surgery. Different explanatory approaches to the occurrence of radiolucencies have been established. Retrieval analysis demonstrated, that radiolucencies of well-fixed unicompartmental prostheses were associated with the histological findings of fibrocartilaginous connective tissue at the cement-bone interface. Decreased primary fixation strength of the prosthesis might lead to increased micromotion of the implant, which facilitates ingrowth of such connective tissue at the interface [11]. Errors in the cementation technique might as well contribute to the occurrence of radiolucencies in cemented OUKA. As a consequence, the cementless OUKA was developed in order to address these problems. Initial studies have shown that the incidence of radiolucent lines is lower in cementless OUKA compared to cemented OUKA with equivalent functional outcomes in the short-term [13]. This might reduce revision rates in OUKA due to a suspected aseptic loosening. On the other hand, the risk for periprosthetic fracture in cementless OUKA has found to be higher compared to cemented OUKA [14] and there is a lack of independent level I and level II studies in order to assess the safety and equivalence of cementless OUKA in comparison to the well-documented clinical results of the cemented OUKA. Therefore, the aim of this study is to investigate the clinical, functional, and radiological results of cemented versus cementless OUKA in a prospective randomized, controlled trial.

\section{Study Design And Setting}

This study has a prospective, randomized, controlled, double-center, non-inferiority design. Patients with anteromedial OA will be randomized to either cementless or cemented unicompartmental knee arthroplasty (UKA).

Patients will be screened and recruited either in the outpatient clinic of the trial coordinating Center for Orthopedics, Trauma Surgery and Spinal Cord Injury, Heidelberg University, Germany or at the Department of Orthopedics and Traumatology at the University Hospital of Linz in Austria. All patients with 
anteromedial OA will be screened and enrolled according to eligibility criteria. Written informed consent is mandatory before inclusion.

A total of 306 patients will be included according to the sample size calculation and adjusted to estimated drop out of $15 \%$ (see section "Statistical Procedures"). Simultaneously, both centers will recruit patients consecutively until the total number of 306 patients is reached. Thus, inclusion will be competitively. We plan to treat 153 patients at the Department of Orthopedics and Trauma Surgery in Linz. The other 153 patients will be treated in the Department of Orthopedics and Traumasurgery in Heidelberg.

All patients will be asked to take part in the subtrial concerning DXA (dual-energy X-ray absorptiometry) measurements of the periprosthetic bone density. Sample size calculation demonstrated that a total number of 150 patients will be needed to participate in the DXA subtrial (75 patients with cementless and 75 patients with cementless UKA, see section "Statistical Procedures"). The first DXA measurement will be performed within 2 weeks before surgery and the second measurement will be performed at 2-year follow-up in order to assess changes in the periprosthetic bone density around the tibial and femoral implants.

The Surgeons who participate in this study are all experienced and well-trained with the cemented and the uncemented version of the OUKA prosthesis.

The duration of the trial for each subject will be 24 months for evaluation of the primary endpoint. Additionally, a long-term follow up will be performed 5 and 10 years after study intervention. The study flow chart (figure 1) illustrates the course of the study.

\section{Primary outcome measures}

The main purpose of the study is to compare clinical and functional results 2 years after cemented versus uncemented unicompartmental knee arthroplasty using the Oxford Knee Score (OKS) as primary outcome parameter.

- Function and pain in daily life after knee arthroplasty as assessed by the Oxford Knee Score (OKS), a 12-item patient reported outcome measure (PROM) referring to a time period within the last 4 weeks

\section{Secondary outcome measures}

- Clinical outcome (pain, stability, alignment and range of motion) and function (walking distance, climbing and descending stairs) as assessed by the American Knee Society Score (AKSS-O and AKSS-F, best result 100 points each)

- Health related quality of life (mobility, self-care, usual activities, pain/discomfort and anxiety/depression) as assessed by the EuroQol EQ-5D questionnaire

- Pain, symptoms, function in daily living, function in sports and recreation, and knee related quality of life as assessed by the Knee Injury and Osteoarthritis Outcome Score (KOOS) 
- Pain as assessed by a visual analogue scale (continuous scale from 0 - no pain - to 10 - worst pain)

- Satisfaction as assessed by a visual analogue scale (continuous scale from 1 - completely satisfied - to 5 - dissatisfied)

- Activity as assed by the score of the University of California (UCLA, selection of 10 possible activity levels, highest level: 10)

- Awareness of the artificial joint as assessed by the Forgotten Joint Score (FJS-12, a 12-item patient reported outcome measure evaluating the awareness of artificial joint in daily activities; 0 - not aware; 100 - mostly aware)

- Radiographic features including signs of implant loosening and radiolucency lines as assessed by fluoroscopically guided radiographs in two planes (weight bearing zones underneath the tibial component on an a.p.-radiograph and the femoral component on a lateral radiograph are divided into 6 zones each, the zones and the frequency of radiolucent lines will be counted)

- Duration of surgery, blood loss and length of hospital stay as assessed from the surgical documentation

- Complications and reoperation rate as assessed during the follow-up visits

In a subtrial, the influence of the fixation technique on periprosthetic bone mineral density (BMD) in cemented versus cementless medial unicompartmental knee arthroplasty as well as possible correlations between $\mathrm{BMD}$ and the incidence of radiolucent lines and/or periprosthetic fractures will be examined in a subgroup of 150 patients of the study cohort.

- The primary outcome measure of the subtrial will be the difference in mean BMD as measured by dual-energy X-ray absorptiometry (DXA) around the femoral and tibial components between the preoperative and postoperative measurements two years after surgery.

\section{Study Participants}

Patients suffering from end stage anteromedial OA of the knee will be screened and considered eligible for medial unicompartmental knee arthroplasty by fulfilling the Oxford UKA criteria (J. W. Goodfellow et al., 1988). These are listed below in the inclusion criteria. Patients will be recruited at the outpatient clinics at the study sites in Heidelberg and Linz.

\section{Inclusion Criteria}

Subjects meeting all of the following criteria will be considered for admission to the trial:

- Anteromedial osteoarthritis with full thickness cartilage loss of the medial compartment

- Well preserved lateral compartment on valgus stress $\mathrm{x}$-ray images

- Passively correctable intra-articular varus deformity

- 18 years of age or older 
- Ability of the subject to understand the character and individual consequences of the clinical trial

- Signed informed consent (must be available before enrollment in the trial)

\section{Exclusion Criteria}

Subjects presenting with any of the following criteria will not be included in the trial:

- Patients found to be unsuitable for Oxford UKA before or during surgery

- Preoperative extension limitation greater than 15 degrees and/or maximal flexion of less than 100 degrees

- Severe lateral patellar facet OA with bone loss and grooving

- Functionally insufficient anterior cruciate ligament

- History of hypersensitivity reaction to metal implants made of cobalt-chromium-alloys

- All forms of inflammatory arthritis (such as rheumatoid arthritis)

- Patients with previous PTO (proximal tibial osteotomy)

- Patients with intraarticular fracture and tibial condyle fracture sequelae

- History of septic arthritis

- Senile dementia

- Insufficient knowledge of the German language (written and spoken)

- Participation in other clinical trials or observation period of competing trials, respectively

- Patient is pregnant or considered a member of a protected population (e.g., prisoner, mentally incompetent, etc.)

\section{Recruitment and Assessment}

Initial screening of the patients will take place in the outpatient clinic of the two centers. Each patient will be evaluated through a surgeon who will perform the intervention at the respective study site. All patients with anteromedial OA of the knee who are scheduled for surgery will receive a patient information sheet with further explanation of the study and, in case of a subtrial participation, with additional information concerning DXA measurements.

Further visits will be designed as follows (see Table 1):

\section{Baseline Visit}

The baseline visit will take place 1 to 5 days prior to surgery in the outpatient clinic. Written informed consent for study participation will be obtained after evaluation of inclusion and exclusion criteria. The questionnaires (OKS, AKS, UCLA, EQ-5D, KOOS, FJS-12, VAS) will be completed and the demographic data, the medical history, diagnosis and relevant concomitant medication will be assessed. 
Additionally, a physical examination will be performed. Routine radiographs (taken in standing position: complete leg a.p., knee in a.p. and lateral view and in a lying position: knee a.p. in varus and valgus stress) will be taken.

Patients who gave written consent will receive a DXA measurements at the femoral neck and the proximal tibia.

\section{Intervention Visit}

After randomization (see section "Randomization") the designated surgeon will implant either a cemented or cementless OUKA (see section "Intervention"). Intraoperative findings including implant sizing, the duration of surgery, the duration of blood arrest/tourniquet closure, the blood loss, whether local periarticular anesthesia was applied, whether perioperative application of tranexamic acid was used, the use of a drainage and which type of skin suture was used, as well as possible intraoperative complications will be documented in detail.

\section{Postoperative Visit (day 7 or discharge Visit)}

After surgery the following assessments will be evaluated: ROM at the last day of hospitalization, duration of hospitalization, documentation of postoperative complications, peri-/postoperative transfusion of red cell concentrates, postoperative blood loss through the drainage, VAS (each day) and evaluation of postoperative radiographs with regard to implant position.

\section{Follow up Visits}

Follow-up visits will be scheduled 3 months, 1 year, 2 years, 5 years and 10 years after surgery. All patients will be informed about their scheduled appointments in the written discharge report and will be contacted via telephone or letter prior the scheduled appointment. During all follow-up visits the following items will be assessed: change in concomitant medication, physical examination (ROM, persisting lymph edema), radiographic evaluation, completion of questionnaires (OKS, AKS, UCLA, EQ-5D, KOOS, FJS-12, VAS), postoperative complications (inlay luxation, periprosthetic fracture, revision surgery due to hematoma, superficial infection of the wound, deep infection of the prosthesis, and any reoperation associated with implant revision) and the second DXA measurement (after 2 years). 


\begin{tabular}{|c|c|c|c|c|c|c|c|c|c|}
\hline sit No. & & Visit 1 & Visit 2 & Visit 3 & Visit 4 & Visit 5 & Visit 6 & Visit 7 & Visit 8 \\
\hline & $\begin{array}{l}\text { relative time } \\
\text { point }\end{array}$ & Baseline & Surgery & $\begin{array}{l}\text { post- } \\
\text { operative }\end{array}$ & $\begin{array}{l}3 \\
\text { months }\end{array}$ & $\begin{array}{l}12 \\
\text { months }\end{array}$ & $\begin{array}{l}24 \\
\text { months }\end{array}$ & 5 years & 10 years \\
\hline & time frame & $\begin{array}{l}\text { Day }-5 \\
\text { to day } \\
-1\end{array}$ & Day 0 & Day 1-7 & $\begin{array}{l} \pm 3 \\
\text { weeks }\end{array}$ & $\begin{array}{l} \pm 1 \\
\text { month }\end{array}$ & $\begin{array}{l} \pm 1 \\
\text { month }\end{array}$ & $\begin{array}{l} \pm 2 \\
\text { months }\end{array}$ & $\begin{array}{l} \pm 2 \\
\text { months }\end{array}$ \\
\hline & $\mathrm{R} / \mathrm{S}^{*}$ & & & & & & & & \\
\hline $\begin{array}{l}\text { clusion/ exclusion } \\
\text { iteria }\end{array}$ & $\mathrm{S}$ & $\bullet$ & $\bullet$ & & & & & & \\
\hline $\begin{array}{l}\text { ritten informed } \\
\text { nsent }\end{array}$ & $\mathrm{s}$ & - & $\bullet$ & & & & & & \\
\hline indomization & $\mathrm{S}$ & & - & & & & & & \\
\hline tervention & & & $\bullet$ & & & & & & \\
\hline lysical examination & $\mathrm{R}$ & $\bullet$ & & $\bullet$ & $\bullet$ & $\bullet$ & $\bullet$ & $\bullet$ & $\bullet$ \\
\hline idiographs & $\mathrm{R}$ & $\bullet$ & & $\bullet$ & $\bullet$ & $\bullet$ & $\bullet$ & $\bullet$ & $\bullet$ \\
\hline$\overline{\mathrm{KA}}$ & $\mathrm{S}$ & $\bullet$ & & & & & $\bullet$ & & \\
\hline lestionnaires & $\mathrm{S}$ & $\bullet$ & & & $\bullet$ & $\bullet$ & $\bullet$ & $\bullet$ & $\bullet$ \\
\hline edical history & $\bar{R}$ & $\bullet$ & & & & & & & \\
\hline mcomitant medication & $\mathrm{R}$ & $\bullet$ & & $\bullet$ & $\bullet$ & $\bullet$ & $\bullet$ & $\bullet$ & $\bullet$ \\
\hline mplication & $\mathrm{R}$ & & - & - & - & - & - & & \\
\hline rious adverse events & & & $\bullet$ & $\bullet$ & & & & & \\
\hline
\end{tabular}

$* \mathrm{R}=$ Regular or $\mathrm{S}=$ study specific examination

\section{Prior and Concomitant Disease/Medication/Treatment}

Relevant additional diseases, medication and treatments present at the time of informed consent are regarded as concomitant diseases and will be documented on the appropriate pages of the case report form (CRF).

Following concomitant diseases/medication and treatment will be documented:

- Diabetes mellitus

- Autoimmune diseases

- History of prior knee surgery

- History of trauma to the affected limp

- Varus or Valgus Deformity on long-leg x-ray (femoro-tibial angle)

The treatment of accompanying illnesses not subject to the exclusion criteria is permissible if this is not expected to have any effect on the outcome measures used in this study.

The following drug groups are permitted under restriction as concomitant medication: 
- immunosuppressive drugs

\section{Randomization}

Consecutively screened and eligible patients will be included in the trial at each center after initiation of the study. In order to achieve comparable intervention groups, patients will be allocated concealed by preoperative randomization using the web-based online randomization tool randomizer.at provided by the Institute of Medical Informatics, Statistics and Documentation of the Medical University of Graz (https://www.randomizer.at). Randomization will only be done by authorized trial personnel with their login data.

\section{Intervention}

Medial unicompartmental knee arthroplasty (UKA) involves only the diseased area of the joint being replaced. The healthy compartment of the knee is retained and artificial implants are inserted in place of the diseased area. This is done via a minimally invasive surgical procedure. The Oxford® Partial Knee (Zimmer Biomet Deutschland GmbH, Berlin, Germany) will be used for unicompartmental medial knee arthroplasty in a cemented and a cementless version as part of this study. Surgery will be performed following the recommendations provided by the company and the Microplasty ${ }^{\circledR}$ Instrumentation will be used. In both groups early weight bearing will be allowed as soon as tolerated.

\section{Safety}

The patients will undergo unicompartmental medial knee arthroplasty as a routine surgical procedure. Patients will be informed of the standard risks associated with anesthesia and knee arthroplasty operation. In addition to routine diagnostic procedures patients participating in the substudy will undergo assessment of bone mineral density via DXA preoperatively and two years after surgery.

Subjects may withdraw their consent at any time without any disadvantage without stating the reason. Patients may also be excluded for other reasons if the investigator assesses a continuation of the clinical trial as detrimental to the subject's well-being.

If serious safety concerns arise, the lead investigator can terminate or interrupt the study. If new information on the risk-to-benefit ratio of the treatment/intervention used in the study is obtained in the meantime, the lead investigator reserves the right to interrupt or terminate the project. Premature termination is also possible if the lead investigator notices and agrees upon that patient recruitment is insufficient and that this cannot be expedited by appropriate measures.

The involvement in the trial will not result in a specific benefit to the patients, although specific outcome measurements may be perceived as a benefit by some patients. Continuous clinical and radiological follow-up as well as the DXA measurement during the study might be beneficial for patients participating in the study because possible complications could be detected at an early stage. The main benefits of the trial will be to future patients by providing information about the best mode of fixation. 
Adverse events will be documented upon occurrence. For the purpose of this study an adverse event (AE) is defined as any untoward medical occurrence in a subject participating in a study and which does not necessarily have a causal relationship with the study treatment. An AE can therefore be any unfavorable and unintended sign, symptom or disease temporally associated with the study treatment.

A pre-existing disease or symptom will not be considered an adverse event unless there will be an untoward change in its intensity, frequency or quality. This change will be documented by an investigator.

AEs will be classified as "serious "(SAE) in case it is life-threatening at the time of the event or results in death, requires inpatient hospitalization or prolongation of existing hospitalization, results in persistent or significant disability/incapacity or other important medical events. All other AEs will be classified as "nonserious".

All SAEs will be documented in the "Serious Adverse Event" form.

The following postoperative complications are defined and will be reported during the study as "Serious Adverse Event":

- superficial infection of the wound

- deep infection of the prosthesis

- revision surgery due to hematoma at the site of operation

- dislocation of the inlay

- periprosthetic fracture

- loosening of the implant

- progression of osteoarthritis of the lateral compartment resulting in revision surgery

- any reoperation due to complications associated with the implant.

A 3-grade scale will be used to classify the AE intensity:

1) Mild AEs can easily be tolerated and symptoms can be ignored or disappear when the subject is distracted; 2) Moderate AEs cause discomfort but are tolerable, cannot be ignored and affect the normal activity; and 3) If symptoms affect the normal activity strongly, the AE will be classified as severe. The investigator will evaluate each $\mathrm{AE}$ that occurred regarding the coherency with the study intervention. The clinical course of the AE will be followed up until resolution of symptoms or until it has changed to a stable condition.

\section{Data Management}

All protocol-required information collected during the trial must be entered by the investigator, or designated representative, in the case report form (CRF). 
The completed CRF must be reviewed and signed by the investigator or by a designated sub-investigator. After keeping a copy at the trial center, the original Case Report Form is sent to the IMBI, University of Heidelberg, being in charge of the data management within the CUPKAT trial.

In order to ensure that the database reproduces the case report forms correctly, the IMBI accomplishes a double entry of data. A tracking system for CRF data and queries will be established to guarantee that data is managed in a timely manner. In order to guarantee high quality of data completeness, validity and plausibility of data as defined in a data validation plan will be checked using validating programs, which will generate queries. The investigator or the designated representatives are obliged to clarify or explain the queries. If no further corrections are to be made in the database it will be closed and used for statistical analysis. All data management procedures will be carried out on validated systems and according to the current standard operating procedures (SOP) of the IMBI.

At the end of the study, the data will be transformed into different data formats (e.g. csv-files) to ensure that it will be possible to reuse it. The principle investigator will retain the originals of all CRFs and the trial data for long-term preservation.

The investigators have to arrange the retention of the subject identification codes for at least 10 years after the completion or termination of the trial. Patient files and other source data shall be kept for the maximum period of time permitted by the hospital.

The lead investigator or other owner of the data shall retain all other documentation pertaining to the trial for at least 10 years. This documentation shall include:

- the protocol including the rationale, objectives and statistical design and methodology of the trial, with conditions under which it is performed and managed, and details of the study intervention,

- standard operating procedures,

- all written opinions on the protocol and procedures,

- final report,

- case report forms,

- audit certificate(s), if available,

- all other relevant documents of the trial master file, according to the ICH-GCP guideline.

Any change of data ownership shall be documented. All data shall be made available if requested by relevant authorities.

The investigator(s) will archive all trial data to local law or regulations.

\section{Monitoring and Audits}

Monitoring is not required however the coordinating Investigator or Zimmer Biomet as the funder of the study, may perform audits to ensure the investigation is being conducted consistent with the protocol and 
recruitment is on target.

\section{Statistical Procedures}

All statistical procedures will be performed and supervised by the IMBI (Institute of Medical Biometry and Informatics, University of Heidelberg). Sample size was calculated for the primary endpoint, the Oxford Knee Score (OKS), and was based on a two-group comparison using Student's t-test for a NI design with a $\mathrm{NI}$ margin of 4 points. Following the recommendation by Beard et al. 2015, a difference of 4 points in means between the randomized groups was considered as clinically important, indicating that any lower value would fall within measurement error. According to Streit et al. 2015 a likely standard deviation for the OKS is 10 points.

Assuming equivalent performance of the two groups for the OKS and a NI margin of 4 points with a power of $1-\beta=90 \%$ using a two-sample t-test at a two-sided significance level $a=5 \%$, a total of 266 patients (133 per arm) will be required.

The sample size calculation was performed using the PROC POWER procedure in SAS 9.4. In order to allow for possible loss to follow-up $15 \%$ more patients will be recruited, the sample size was adjusted to a total of 306 patients (153 per arm).

In the event that the $\mathrm{NI}$ hypothesis is met and successful, the Oxford cementless arm will be tested for superiority. If the lower $95 \%$ confidence interval on the difference of Oxford Cementless OKS minus Oxford Cemented OKS exceeds zero (0), superiority of Oxford cementless UKA over Oxford cemented UKA will be claimed.

For the subtrial, a sample size of 75 patients per group $(n=150)$ will provide sufficient power to show differences in terms of BMD $>10 \%$ as statistically significant [Hooper et al. BJJ 2013].

The primary analysis will be performed for the full analysis set which comprises all patients randomized into the trial. In this set, every patient is analyzed according to the group randomized into (intention to treat principle).

The per-protocol set will comprise all patients who were treated according to the randomized treatment. Before the study is evaluated, rules for selecting the per-protocol set will be selected by a steering board with at least the lead investigator and the study statistician as members.

The safety set will comprise all patients who have received study intervention, and will allocate the patients to the intervention they actually received, regardless of randomization.

The primary analysis will be performed by testing the following NI hypothesis: Mean OKS plus NI margin (4 points) after 1 and 2 years in cementless UKA is higher than mean OKS in cemented UKA. This leads to the following hypotheses: 
$\mathrm{HO}: \mu_{\text {cemented }}>\mu_{\text {cementless }}+4$ points

$\mathrm{H} 1: \mu_{\text {cemented }}<\mu_{\text {cementless }}+4$ points

This hypothesis will be tested at the $a=0.05$ level using a two-factorial analysis of covariance (ANCOVA) with intervention and gender as factors and BMI, OKS pre-operative, and VAS pre-operative as covariables. Additionally, the primary endpoint will be evaluated in a multivariable linear regression model with OKS as the response and intervention, age, gender, BMI, OKS pre-operative, and VAS pre-operative as additional explanatory variables. We will examine the residual to assess model assumptions and goodness-of-fit. Secondary analyses will be performed on the variables according to the secondary outcome measures. We will use a chi-squared test for categorical variables, and a t-test for continuous variables. All scores in the set of secondary endpoints will be examined as total score, subscales will not be analyzed.

For DXA-subgroup analysis on the primary variable we consider the following groups:

- Age $<60$ years vs. age $>60$ years

- Male vs. female

- Complete cartilage damage ("bone on bone") vs. incomplete cartilage damage (not "bone on bone")

- Tibial varus angle of greater than 15 degrees

The first statistical analysis and report is planed 6 months after last subject out (LSO, approximately 07/2023) for the primary endpoint.

All information concerning the trial is confidential before publication. Publication(s) and/or presentation(s) of the study results is encouraged after appropriate time for review and written agreement by the lead investigator. The lead investigator as well as the Funder of the study have to be provided with a draft of the abstract and/or manuscript for review and editorial comments at least 30 days prior to submission and/or presentation. The lead investigator does not have the right to prevent publication, except for patent or copyright reasons.

\section{Trial Status}

Protocol Version V03 dated 25.09.2019.

Ethics approval has been granted. The trial has been registered. All necessary documents concerning the study have been finalized. Estimated start of patient recruitment will be $07 / 2020$ with estimated completion 07/2021.

\section{Abbreviations}


AE: Adverse Event; BMD: Bone Mineral Density; DXA: Dual-energy X-ray absorptiometry; OA: Osteoarthritis; FSI: First Subject In; LSI: Last Subject In; LSO: Last Subject Out; OKS: Oxford Knee Score; OUKA: Oxford Unicompartmental Knee arthroplasty; UKA: Unicompartmental Knee arthroplasty; VAS: Visual Analog Scale (pain)

\section{Declarations}

\section{Ethics approval and consent to participate}

The study will be performed in accordance with the 1964 Helsinki declaration and its later amendments and is approved by the Ethics Commission of the Medical Faculty of the University of Heidelberg (S685/2017). Written informed consent will be obtained from every individual participant before study enrolment.

\section{Consent for publication}

not applicable

\section{Availability of data and material}

The data that support the findings of this study are available from the corresponding author, TR, upon reasonable request.

\section{Competing interests}

Author BP, TR, TB and ASG have no conflicts of interests to declare. Authors CM and TG report research funding and paid lectures from Zimmer Biomet outside the submitted work.

\section{Funding}

The study is financially supported by a research fund by Zimmer Biomet Deutschland $\mathrm{GmbH}$, Merzhauser Str. 112, 79100 Freiburg. Breisgau, Germany; grant number 120,000 €.

The funding Company was neither involved in the design of the study nor the collection, management, analysis, interpretation of data and writing the manuscript.

\section{Authors' contributions}

Chief Investigator CM; Conceptualization BP, TR, TG and CM.; Methodology, BP, TR, TB, ASG, CM; Project administration, TR, BP; Supervision, TR, BP, TG and CM Visualization BP; Radiological Evaluation of the DXA-Sub Trial CR, Writing - original draft, BP Writing - review \& editing, BP, TR, TG, CR, TB, ASG and CM; statistical evaluation TB; Data Management ASG

\section{Acknowledgements}


Financing of the CUPKAT study was granted by Zimmer Biomet Deutschland GmbH.

The Lead Investigator is Professor Christian Merle. The two centers which take part in the study will be the Center for Orthopedics, Trauma Surgery and Spinal Cord Injury, Heidelberg University, Germany (Coordinating Center) and the Department for Orthopedics and Traumatology, Kepler University Hospital $\mathrm{GmbH}$, Linz, Austria.

The steering committee consists of Professor Christian Merle, Professor Tobias Gotterbarm, Dr Benjamin Panzram and Dr Tobias Reiner.

Planning and organization of the trial was supported and supervised by the Coordination Centre for Clinical Studies (KKS, Heidelberg University), Dr Steffen Lunz and Andrea Seidel-Glätzer.

Evaluation of the DXA measurement in the subtrial, Dr Christoph Rehnitz

Data monitoring and management as well as statistical procedures will be carried out by the IMBI (Institute of Medical Biometry and Informatics, Professor Mainhard Kieser, Heidelberg University)

We would like to thank all mentioned participants and their teams for the support throughout the planning and preparation of the study.

\section{Additional Files}

SPIRIT guidance checklist. PDF file.

\section{References}

1. Isaac, S.M., et al., Does arthroplasty type influence knee joint proprioception? A longitudinal prospective study comparing total and unicompartmental arthroplasty. Knee, 2007. 14(3): p. 212-7.

2. Murray, D.W., J.W. Goodfellow, and J.J. O'Connor, The Oxford medial unicompartmental arthroplasty: a ten-year survival study. J Bone Joint Surg Br, 1998. 80(6): p. 983-9.

3. Campi, S., et al., Ten-year survival and seven-year functional results of cementless Oxford unicompartmental knee replacement: A prospective consecutive series of our first 1000 cases. Knee, 2018. 25(6): p. 1231-1237.

4. Mohammad, H.R., et al., Long-term outcomes of over 8,000 medial Oxford Phase 3 Unicompartmental Knees-a systematic review. Acta Orthop, 2018. 89(1): p. 101-107.

5. Australian Orthopaedic Association National Joint Replacement Registry (AOANJR). Hip, Knee \& Shoulder Arthroplasty: 2018 Annual Report. Adelaide: AOA, 2018.

6. Lewold, S., et al., Revision of unicompartmental knee arthroplasty: outcome in 1,135 cases from the Swedish Knee Arthroplasty study. Acta Orthop Scand, 1998. 69(5): p. 469-74. 
7. Robertsson, O., et al., Knee arthroplasty in Denmark, Norway and Sweden. A pilot study from the Nordic Arthroplasty Register Association. Acta Orthop, 2010. 81(1): p. 82-9.

8. van der List, J.P., et al., Outcomes of cementless unicompartmental and total knee arthroplasty: A systematic review. Knee, 2017. 24(3): p. 497-507.

9. National Joint Registry for England Wales Northern Ireland and the Isle of Man, et al. National Joint Registry for England, Wales, Northern Ireland and the Isle of Man: 14th Annual Report. 2017.

10. The New Zealand Joint Registry, et al. The New Zealand Joint Registry - Eighteen Year Report, January 1999 to December 2016. 2017.

11. Kendrick, B.J., et al., Histology of the bone-cement interface in retrieved Oxford unicompartmental knee replacements. Knee, 2012. 19(6): p. 918-22.

12. Pandit, $\mathrm{H}_{\text {., }}$ et al., Improved fixation in cementless unicompartmental knee replacement: five-year results of a randomized controlled trial. J Bone Joint Surg Am, 2013. 95(15): p. 1365-72.

13. Pandit, $\mathrm{H}$., et al., Cementless Oxford unicompartmental knee replacement shows reduced radiolucency at one year. J Bone Joint Surg Br, 2009. 91(2): p. 185-9.

14. Liddle, A.D., et al., Cementless fixation in Oxford unicompartmental knee replacement: a multicentre study of 1000 knees. Bone Joint J, 2013. 95-B(2): p. 181-7.

\section{Figures}



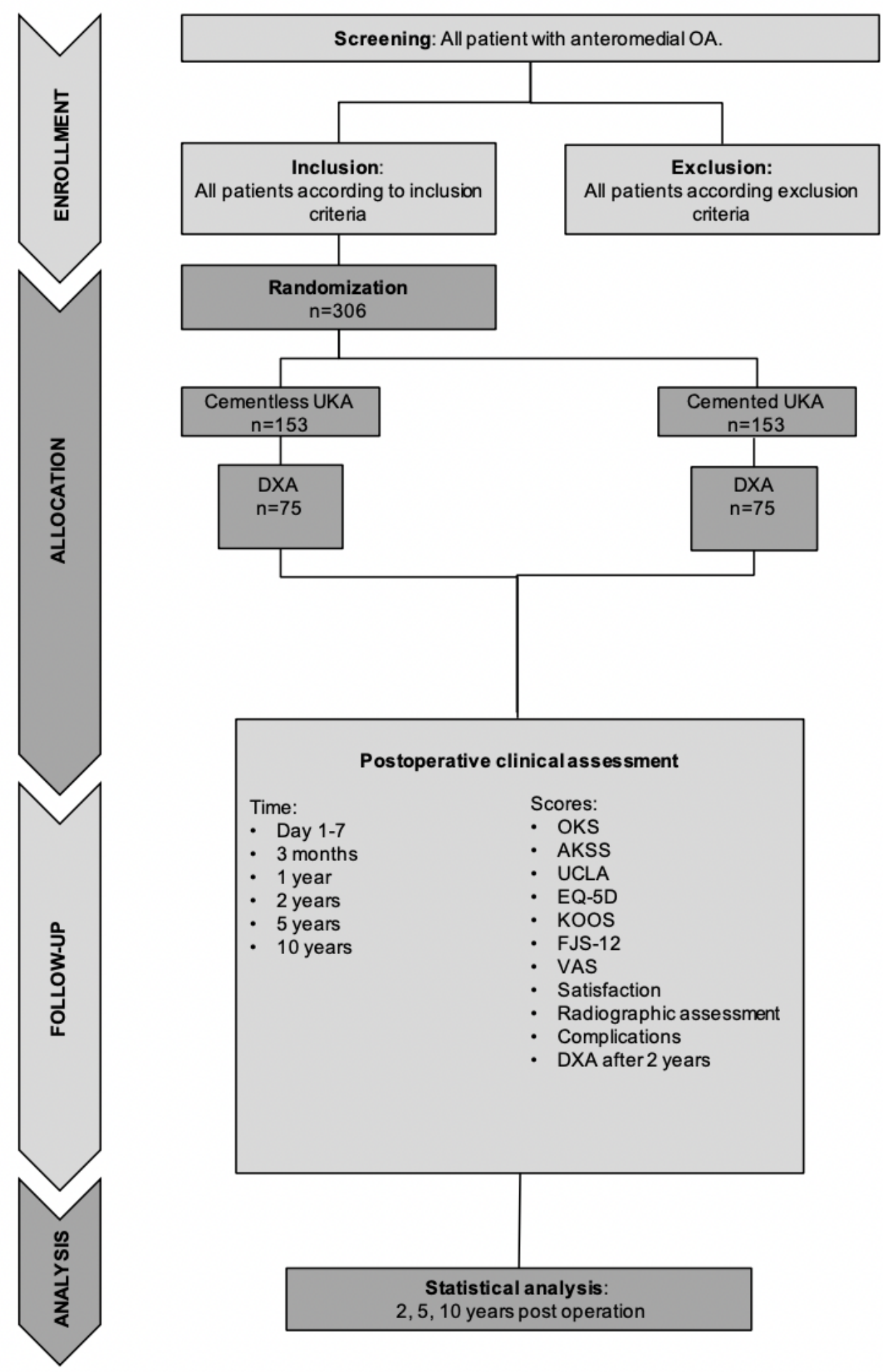

Figure 1

Study flow chart illustrating the course of the study.

\section{Supplementary Files}

This is a list of supplementary files associated with this preprint. Click to download. 
- SPIRITFillablechecklistcompleted.doc 